\title{
Family Physicians Increasingly Deliver Care in Diverse Languages
}

\author{
Aimee R. Eden, PhD, MPH, Andrew Bazemore, MD, MPH, Zachary J. Morgan, MS, and \\ Yalda Jabbarpour, $M D$
}

The proportion of family physicians reporting provision of patient care in Spanish changed little between 2013 to 2020 but rose substantially for care delivered in other non-English languages. Physician-patient language concordance is associated with better clinical outcomes and higher patient satisfaction, serves as a proxy indicator for workforce diversity, and should be monitored and encouraged as the US population continues to diversify. ( $\mathrm{J}$ Am Board Fam Med 2022;35:5-6.)

Keywords: Diversity, Family Medicine, Family Physicians, Language, Limited English Proficiency, Linguistics, Patient Care, Patient Satisfaction, Physician-Patient Relations, Primary Health Care, Workforce

Linguistic diversity in the US population is growing, drawing increased attention to language-concordant clinical care, particularly in its largest and most widely distributed delivery platform, primary care. ${ }^{1}$ Patients with limited English proficiency experience worse outcomes related to language barriers, leading to health disparities. ${ }^{1,2}$ Language-concordant care for patients with limited English proficiency has been shown, in most cases, to improve patient outcomes and patient satisfaction, ${ }^{2,3}$ so a linguistically diverse family physician workforce has important population health implications and may contribute to reducing health disparities. Our objective was to track the frequency of self-reported provision of primary care in Spanish and other languages over time to understand trends in patient-physician language concordance.

This article was externally peer reviewed.

Submitted 3 May 2021; revised 29 June 2021; accepted 6 July 2021.

From American Board of Family Medicine, Lexington, KY (ARE, AB, ZJM); Robert Graham Center, Washington, DC (YJ).

Funding: none.

Conflict of interest: Drs. Bazemore and Eden and Mr. Morgan are employees of the American Board of Family Medicine.

Corresponding author: Aimee R. Eden, PhD, MPH, 1648 McGrathiana Parkway, Suite 550, Lexington, KY 40511 (E-mail: aeden@theabfm.org).

\footnotetext{
See Related Commentary on Page 200.
}

We used data collected from 2013 through 2020 on the American Board of Family Medicine (ABFM) Family Medicine Certification Examination registration questionnaire, which has a $100 \%$ response rate. ${ }^{4}$ From 2013 to 2016, all certified practicing family physicians registering for certification were asked, "Do you use a language other than English to interact with patients?" and from 2017 to 2020, "Do you personally provide care in a language other than English?" Response options from 2013 to 2020 included "Yes, Spanish," "Yes, Other" (with an open text box), and "No." We calculated frequencies by year to examine changes over time and performed Cochran-Armitage trend tests for both Spanish and other languages by year. Fewer physicians were sampled in 2020 due to both a prior change in the continuous certification process and the extension of certification deadlines due to COVID-19. The American Academy of Family Physicians Institutional Review Board approved this study.

Using data from a total of 64,468 family physicians over the 8 -year period, we found that approximately $22 \%$ of family physicians consistently reported providing care in Spanish $(P=.0203)$, while the proportion that reported providing care in languages other than English or Spanish has increased incrementally and significantly from $7.6 \%$ in 2013 to $15.8 \%$ in $2020(P<.0001)$ (see Figure 1). Depending on how the question was interpreted, it is possible that some physicians who report providing care in a different language do not do so with fluency or even 
Figure 1. Trends in self-reported family physician provision of care in languages other than English, 2013 to 2020.

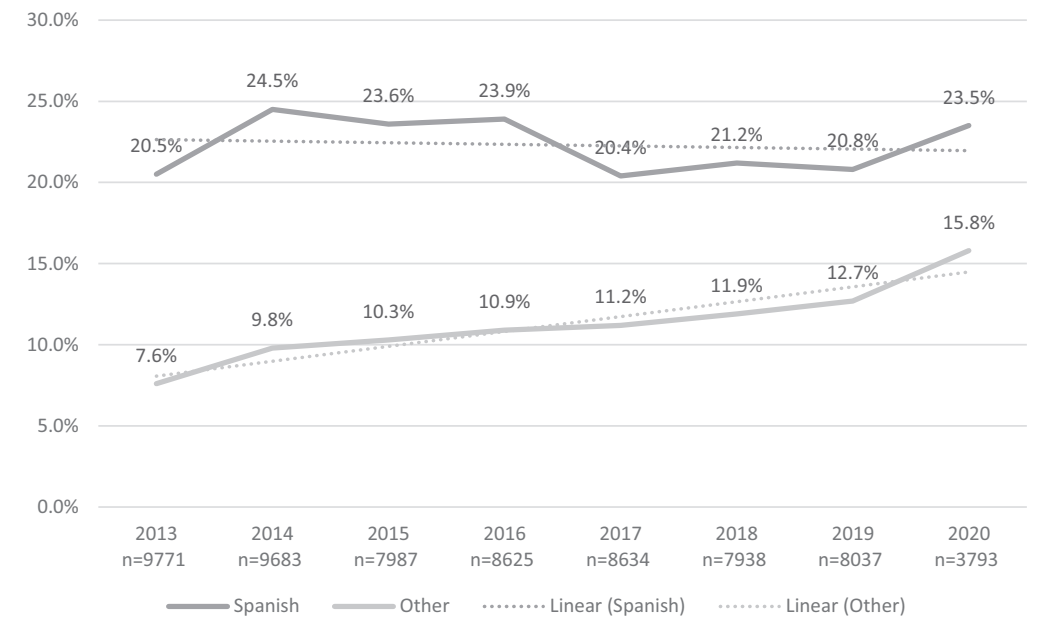

proficiency or may use an interpreter, so it is possible that these findings are overstated.

While the use of trained medical interpreters has been shown to improve the quality of patient care, ${ }^{2,3}$ and physicians are encouraged to provide languageassistance services, ${ }^{5}$ direct communication with a language-concordant provider can improve patient experience, trust, and overall quality of care. ${ }^{1,6}$ As the US population becomes more diverse, the intentional nurturing of the linguistic diversification of the primary care physician workforce through training in medical Spanish and other languages ${ }^{6,7}$ and through attracting and retaining a more diverse workforce will be important elements in moving toward the equitable delivery of health care for patients with limited English proficiency.

\section{References}

1. Diamond L, Izquierdo K, Canfield D, et al. A systematic review of the impact of patient-physician nonEnglish language concordance on quality of care and outcomes. J Gen Intern Med 2019;34:1591-606.
2. Hsueh L, Hirsh AT, Maupomé G, Stewart JC. Patient-provider language concordance and health outcomes: a systematic review, evidence map, and research agenda. Med Care Res Rev 2021;78:3-23.

3. Ngo-Metzger Q, Sorkin DH, Phillips RS, et al. Providing high-quality care for limited English proficient patients: the importance of language concordance and interpreter use. J Gen Intern Med 2007;22:324-30.

4. Peterson L, Fang B, Phillips R, Avant R, Puffer J. A certification board's tracking of their specialty: the American Board of Family Medicine's data collection strategy. J Am Board Fam Med 2019;32:89-95.

5. U.S. Department of Health and Human Services Office of Minority Health [Internet]. National standards for culturally and linguistically appropriate services (CLAS) in health and health care; n.d. [cited 2021 June 14]. Available from: https://thinkculturalhealth.hhs.gov/assets/ pdfs/EnhancedNationalCLASStandards.pdf.

6. Molina RL, Kasper J. The power of language-concordant care: a call to action for medical schools. BMC Med Educ 2019;19:378.

7. Ortega P. Spanish language concordance in US medical care: a multifaceted challenge and call to action. Acad Med 2018;93:1276-80. 